\title{
Role for HIF-1 $\alpha$ and Downstream Pathways in Regulating Neuronal Injury after Intracerebral Hemorrhage in Diabetes
}

\author{
Zhen Yu $u^{a}$ Ling Tang ${ }^{b}$ Lifen Chen ${ }^{a}$ Jinfang Li ${ }^{a}$ Wanfu Wu Changlin $\mathrm{Hu}^{\mathrm{a}}$
}

aDepartment of Neurology, Chongqing University of Medical Sciences, the Second Affiliated Hospital, Chongqing, bDepartment of Neurology, Chongqing University of Medical Sciences, the University-Town Affiliated Hospital, Chongqing, 'Department of Neurology, Chongqing University of Medical Sciences, the First Affiliated Hospital, Chongqing, China

\section{Key Words}

Intracerebral hemorrhage $\cdot H I F-1 \alpha \cdot V E G F \cdot$ Diabetes

\begin{abstract}
Background/Aims: HIF-1 $\alpha$ is accumulated in the cellular nucleus and cytoplasm under conditions of oxygen deprivation and engaged in pathophysiologic changes of homeostasis by modulating the expression of several target genes. As an endogenous signaling protein, HIF-1 $\alpha$ contributes to in neuroprotection, erythropoiesis, and apoptosis modulation. The purpose of this study was to examine the role played by HIF-1 $\alpha$ in regulating neurological injury evoked by intracerebral hemorrhage (ICH) through its downstream product, namely vascular endothelial growth factor (VEGF). In particular, we examined the effects of diabetic hyperglycemia on HIF-1 $\alpha$ response in the processing of ICH. Methods: ELISA was used to measure HIF- $1 \alpha$ and VEGF; and Western Blot analysis to examine the protein expression of VEGFR-2 and Caspase-3. Neurological Severity Score and brain water content were used to indicate neurological function and brain edema. Results: HIF- $1 \alpha$ and VEGF were significantly increased in the brain after induction of ICH in non-diabetic control rats and diabetic rats; however, the amplified levels of HIF-1 $\alpha$ and VEGF were attenuated in diabetic rats $(P<0.05$ $v s$. non-diabetic rats) as compared with non-diabetic rats. Also, the protein expression of VEGF receptor subtype 2 was significantly less in the brain of diabetic rats $(P<0.05$ vs. nondiabetic rats). Further, cerebral infusion of HIF-1 activator stabilized VEGF levels, attenuated Caspase- 3 and improved neurological deficits induced by ICH and the effects are smaller in diabetic animals. Conclusion: HIF- $1 \alpha$ activated by ICH likely plays a beneficial role via VEGF mechanisms and response of HIF- $1 \alpha$ is largely impaired in diabetes. This has pharmacological implications to target specific HIF- $1 \alpha$ and VEGF pathway for neuronal dysfunction and vulnerability related to $\mathrm{ICH}$.

Dr. Ling Tang

and Dr. Zhen Yu

Department of Neurology, The University-Town Affiliated Hospital, Chongqing University of Medical Sciences, 5 University Town Road, Chongqing 400010 (China); and Department of Neurology, The Second Affiliated Hospital, Chongqing University of Medical Sciences, 76 Linjiang Street, Chongqing 400010 (China); E-Mail tangling6463@163.com and yuzheng78@sina.com
\end{abstract}

KARGER 125 


\section{Cellular Physiology Cell Physiol Biochem 2015;37:67-76 \\ \begin{tabular}{l|l|l}
\cline { 2 - 2 } DOI: 10.1159/000430334 & C 2015 S. Karger AG, Basel
\end{tabular} \\ \begin{tabular}{l|l} 
and Biochemistry Published online: August 12, 2015 & www.karger.com/cpb
\end{tabular} \\ Yu et al.: HIF-1 $\alpha$ Activated by Intracerebral Hemorrhage in Diabetes}

\section{Introduction}

In general, intracerebral hemorrhage (ICH) is caused by brain trauma and considered as the second most common cause of stroke because it occurs spontaneously in hemorrhagic stroke. Non-traumatic ICH is also observed to lead to a spontaneous bleeding into the brain tissue and cause a high morbidity and mortality [1]. A number of pathophysiological changes are observed after ICH. i.e., brain edema, blood clotting and neurological deficits [2, 3]. There are many risk factors to be related to non-traumatic ICH. Hyperglycemia is one of many important causes of ICH [4]. It has been reported that patients with diabetes have a greater risk to suffer ICH and have the worse outcomes after ICH than non-diabetics [5-7]. Animal studies further suggest that multiple pathways such as oxidative stress and inflammation etc. are likely involved in the worsened neurological deficits in diabetes after ICH $[8,9]$. However, the mechanisms responsible for ICH that is observed in diabetic patients remain to be determined.

As an important endogenous signaling protein, hypoxia inducible factor-1 (HIF-1) has been reported to play a role in regulating physiologic changes of homeostasis under conditions of oxygen deprivation [10-13]. Elevated subunit HIF-1 $\alpha$ in the cellular nucleus and cytoplasm further regulates the expression of several target genes that are engaged in neuroprotection, erythropoiesis, and apoptosis modulation in protecting tissues against hypoxic conditions [14-17]. Furthermore, inadequate oxygen supply likely appears in the brain regions while ICH occurs and abnormalities of HIF- $1 \alpha$ are also observed in diabetes.

Vascular endothelialgrowth factor(VEGF) is anotherimportantsignalingprotein engaged in both vasculogenesis and angiogenesis, which lead to restoring the oxygen supply to tissues [18]. It has been shown that VEGF stimulates endothelial cell mitogenesis and cell migration, but also enhances microvascular permeability [18]. In addition, it has been reported that VEGF is up-regulated after the ICH and this likely improves the permeability of blood-brain barrier, reduces brain edema formation and promotes the recovery of brain injuries $[19,20]$.

Thus, our current study was to examine the role for HIF- $1 \alpha$ and its downstream pathway product VEGF in regulating ICH-evoked brain injury in diabetic rats and non-diabetic rats. We hypothesized that ICH increases the levels of HIF- $1 \alpha$ and VEGF in the brain tissues and enhancement of HIF- $1 \alpha$ and VEGF is impaired in diabetic animals. This also leads to downregulated protein expression of VEGF receptor subtype 2 (VEGFR-2) and upregulated Caspase-3 (an indicator of cell apoptosis) in diabetic rats as compared with non-diabetic rats. We further hypothesized that cerebral injection of HIF-1 activator improves the levels of VEGF/VEGFR-2, Caspase-3 and brain injury induced by ICH, but the effects are less in diabetic animals.

\section{Materials and Methods}

\section{Animal}

All the animal procedures of this study and their care were conducted in conformity with the guidelines of Institutional Animal Care \& Use Committee (IACUC) of Zhongqing University of Medical Sciences, which are in compliance with the Guideline for the Care and Use of laboratory Animals of the U.S. National Health Institute. The IACUC specifically approved this study. A total of 138 male Sprague-Dawley rats (200-300g) were used in our experiments and all animals were maintained under $12 \mathrm{~h}$ light/dark-cycle with free access to food and water in a temperature- and humidity-controlled room. At the end of each experiment, overdose isoflurance was given to sacrifice the animals.

Induction of Diabetes

Streptozotocin (STZ) was freshly dissolved in $0.9 \%$ sterile saline and diabetes was induced by a single injection of STZ (70 mg/kg i.p., Sigma Co.) as described previously [21]. Diabetes was confirmed by 


\section{Cellular Physiology Cell Physiol Biochem 2015;37:67-76

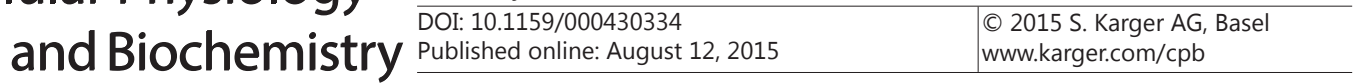 \\ Yu et al.: HIF-1 $\alpha$ Activated by Intracerebral Hemorrhage in Diabetes}

measurements of blood glucose concentrations in samples obtained from the tail vein 3 weeks after injection of STZ. Rats whose blood glucose concentration was $>350 \mathrm{mg} / \mathrm{dl}$ were included in the study. Age- and body weight-matched rats with saline injection were used as controls of non-diabetes. Thus, in this report, STZ rats and non-STZ rats/control rats were named as diabetic rats and non-diabetic rats, respectively.

\section{Induction of ICH}

Control rats and STZ-rats were anesthetized with chloral hydrate (40 mg/kg body weight, i.p.), then immobilized in a stereotaxic apparatus (David Kopf, USA). We used chloral hydrate due to our experiencing in using it and availability. After making a midline incision, the skull was exposed and one burr hole was drilled. Bacterial collagenase (type VII; Sigma Co.) was injected into the right basal ganglia (coordinates, 0.2 $\mathrm{mm}$ anterior, $6 \mathrm{~mm}$ ventral, and $3 \mathrm{~mm}$ lateral to the bregma). Collagenase [0.5 U in $2 \mu \mathrm{l}$ artificial cerebrospinal fluid (aCSF)] was infused into the brain over a period of $5 \mathrm{~min}$ via a Harvard pump before the injection needle was removed and the burr hole was covered by dental zinc cement. The same volume of aCSF was infused into the right basal ganglia as control.

In order to chronically infuse drugs into the brain tissues, animals were cannulated with an L-shaped stainless steel cannula aimed at the right lateral ventricle (coordinates: $3.7 \mathrm{~mm}$ posterior to the bregma, $4.1 \mathrm{~mm}$ lateral to the midline, and $3.5 \mathrm{~mm}$ under the dura). The guide cannula was fixed to the skull using dental zinc cement and jewelers' screw. The cannula was then connected to an osmotic minipump (Alzet pump brain infusion kit, DURECT Inc., Cupertino, CA) with polycarbonate tubing. The pumps were placed subcutaneously between the scapulae, and loaded with vehicle (aCSF) as control or HIF-1 activator

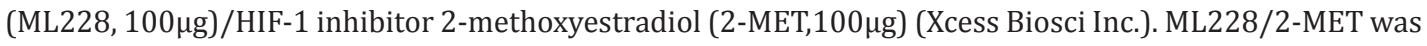
delivered at $0.25 \mu \mathrm{l}$ per hour (Alzet Model 1003D). This intervention allowed animals to receive continuous intracerebroventricular (ICV) infusion via the minipumps before brain tissues were removed.

Thus, rats were divided into four groups. Group 1: non-diabetes/non-ICH; group 2: diabetes/nonICH; group 3: non-diabetes/ICH; and group 4: diabetes/ICH. After completion of those treatments, all the animals were anesthetized and sacrificed 0, 1, 3 and 7 days after ICH for the experiment to examine a time course of HIF- $1 \alpha$ and VEGF responses. In the experiments examining the role of HIF-1 $\alpha$ and VEGF, animals were sacrificed 3 days after ICH. The brains were removed to confirm the placement of the cannula, and the brain regions were dissected under an anatomical microscope and tissues were taken for the process.

\section{ELISA Measurements}

The levels of HIF-1 $\alpha$ were determined using an ELISA assay kit (Abcam Co.) according to the provided description and modification. Briefly, polystyrene 96-well microtitel immunoplates were coated with affinity-purified polyclonal rabbit anti-HIF-1 $\alpha$ antibody. Parallel wells were coated with purified rabbit IgG for evaluation of nonspecific signal. After overnight incubation at room temperature and 2 hours of incubation with the coating buffer containing $50 \mathrm{mM}$ carbonate buffer (pH 9.5) in 2\% BSA, plate were washed with $50 \mathrm{mM}$ Tris- $\mathrm{HCl}$. After extensive washing, the diluted samples and the HIF-1 $\alpha$ standard solutions were distributed in each plate and left at room temperature overnight. The plates were washed and incubated with anti-HIF-1 $\alpha$ galactosidase. Then, the plates were washed and incubated with substrate solution. After an incubation of 2 hours at $37^{\circ} \mathrm{C}$, the optical density was measured using an ELISA reader. This method was also employed to examine the levels of VEGF according to the provided description and modification (Promega Corp. Madison, WI).

\section{Western Blot Analysis}

The brain tissues were removed, homogenized, centrifuged and incubated with streptavidin beads. Streptavidin was used to minimize steric hindrance of the subsequent binding of antigen to the antibody and optimize results of Western blot. The beads were washed and precipitated by centrifugation; sample buffer was added to the collected beads. Beads were pelleted again by centrifugation to obtain supernatant. The supernatant was then diluted to the same volume and applied to SDS-PAGE. Membranes were incubated with the rabbit anti-VEGFR-2 (1:500) and rabbit anti-Caspase-3 primary antibodies (1:1000) and goat anti-rabbit secondary antibody (1:500). Immunoreactive proteins were detected by enhanced chemiluminescence. The membrane was processed to detect $\beta$-actin for equal loading. The densities of protein bands were analyzed using the Scion image software.

\section{KARGER}


Neurological Function and Brain Edema

The modified method of Neurological Severity Score (mNSS) was used to examine neurological function 3 days after treatment. It is noted that mNSS was generally used to assess a combination of motor, sensory, and balance functions [9, 22]. Neurological function was graded on a scale of 0-18 (normal score, 0 ; maximal deficit score, 18). The rats with an abnormal mNSS (score $>0$ ) before ICH were excluded from the experiment.

Brain edema (brain water content) was determined 3 days after ICH. The brain slices (2 mm thick) of the contralateral and ipsilateral hemispheres and cerebellum were cut. The whole brain water content was calculated from all slices $[9,22]$. The brain slices were weighed to obtain the wet weight immediately and dried in an oven at $100{ }^{\circ} \mathrm{C}$ for $24 \mathrm{hrs}$ to obtain the dry weight. The cerebellum was used as the internal control. The water content was expressed as the following formula: [(wet weight) - (dry weight)]/(wet weight) $\times 100 \%$.

\section{Statistical Analysis}

We used a two-way repeated-measures analysis of variance to analyze all the data of time courses for responses of HIF- $1 \alpha$ and VEGF evoked by ICH in this report since the data were obtained from 4 groups at different times. A one-way repeated-measures analysis of variance was used to analyze other data accordingly. Values were presented as means \pm standard error of mean (SEM). Differences were considered significant at $P<0.05$. All statistical analyses were conducted by using SPSS for Windows version 13.0 (SPSS, USA).

\section{Results}

\section{General Measurements}

Figure 1 shows that rats developed hyperglycemia 3 weeks after STZ injection. This figure further demonstrates that an increase in body weight was significantly attenuated in STZ rats ( $\mathrm{n}=82, P<0.05$ vs. control rats) as compared with non-STZ rats $(\mathrm{n}=56)$.

\section{Levels of HIF-1 $\alpha$ and VEGF}

Figure 2 shows that the levels of HIF- $1 \alpha$ were significantly increased in the brain tissues of non-STZ rats and STZ rats 1,3 and 7 days after induction of ICH $(P<0.05$, control rats vs. ICH rats; and STZ rats vs. STZ rats with ICH, $\mathrm{n}=10-12$ in each group). A greater increase was observed 3 days after ICH. Note that the response of HIF-1 $\alpha$ was attenuated in STZ rats compared with non-STZ rats after ICH.

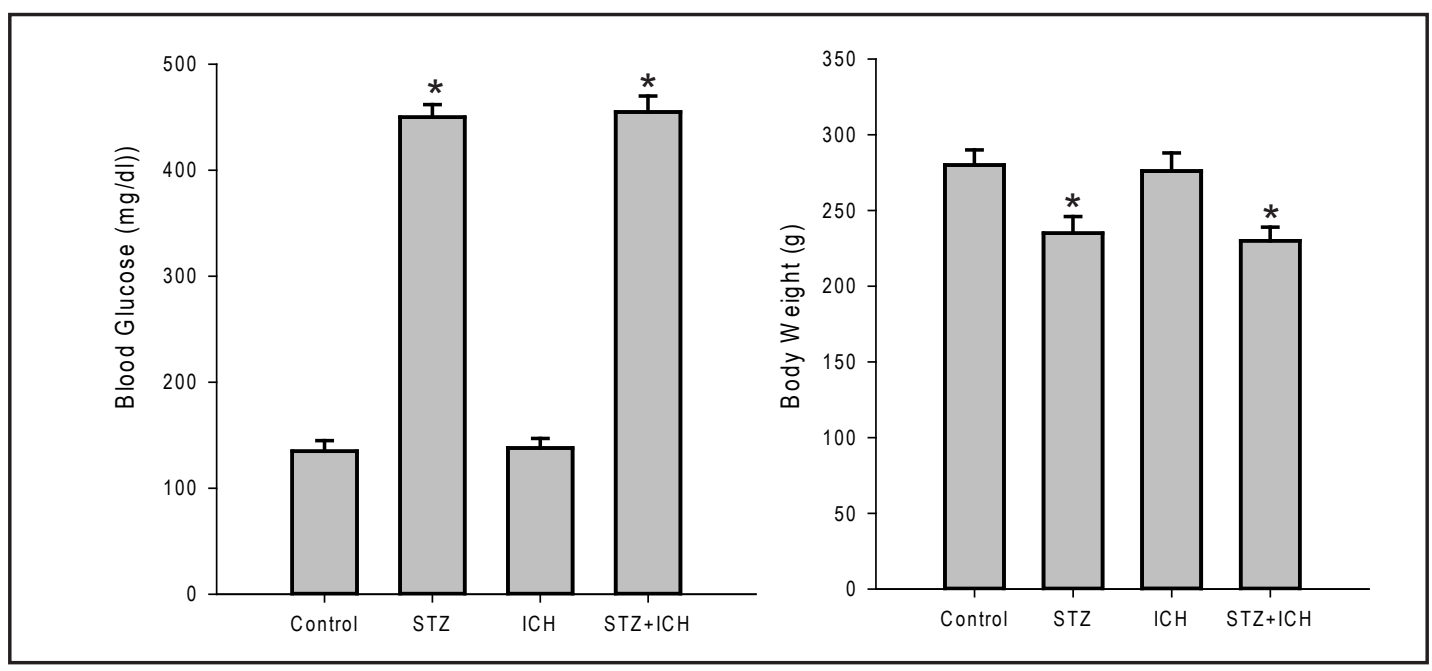

Fig. 1. Effects of STZ injection on blood glucose and body weight. Hyperglycemia was induced in rats 3 weeks after STZ injection and body weight was larger in control rats than that in STZ-rats. Data are expressed as mean \pm SEM. ${ }^{*} P<0.05$, indicated STZ-rats $(\mathrm{n}=56)$ vs. control rats $(\mathrm{n}=82)$. 
Fig. 2. The levels of HIF-1 $\alpha 0,1,3$ and 7 days after induction of ICH. HIF- $1 \alpha$ was significantly increased in non-STZ rats and STZ rats 1,3 and 7 days after induction of ICH. Data are expressed as mean \pm SEM. ${ }^{*} P<0.05$, indicated control rats vs. ICH rats; and STZ rats vs. STZ rats with ICH $(n=10-12$ in each group). $\dagger P<0.05$ vs. non-STZ rats with ICH.

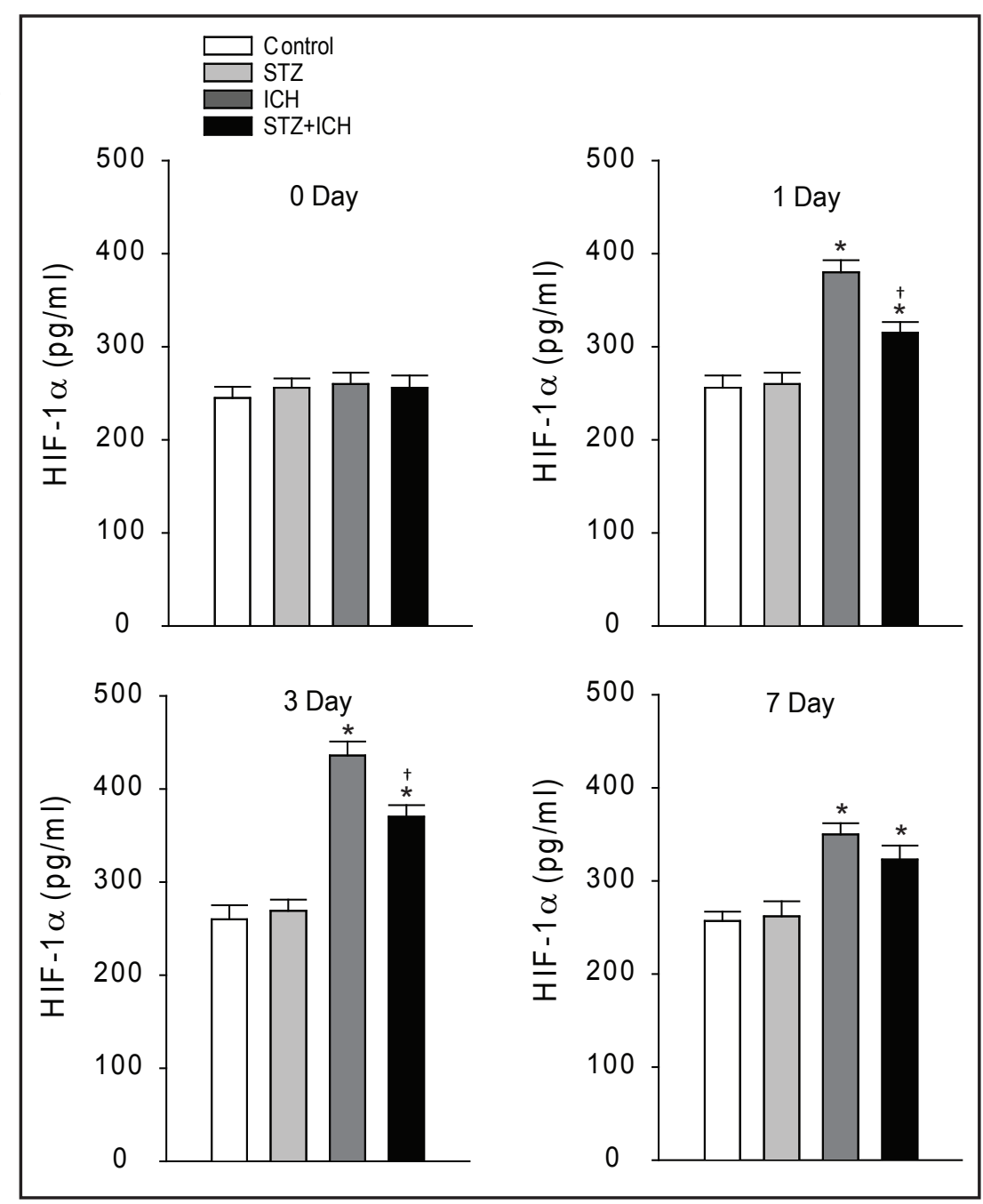

Likewise, Fig. 3 demonstrates that ICH significantly elevated the levels of VEGF in the brain tissues of non-STZ rats and STZ rats $(P<0.05$, control rats vs. ICH rats; and STZ rats $v s$. STZ rats with ICH, $n=10-12$ in each group). The increases were seen 1, 3 and 7 days after induction of ICH and greater increases appeared 3 days after ICH. The similar time courses for responses of HIF-1 $\alpha$ and VEGF evoked by ICH were observed in non-STZ rats and STZ rats. Also, the response of VEGF was attenuated in STZ rats compared with non-STZ rats after ICH.

\section{Effects of 2-MET and ML228 on VEGF}

In this subset of experiments, we examined the effects of 2-MET and ML228 on the levels of VEGF after their ICV administration in order to obtain their effectiveness at the dosage used in this study. Fig. 4 demonstrates that VEGF failed to increase after ICV infusion of 2-MET into the brain of non-STZ rats and STZ rats with ICH $(P>0.05$, control rats vs. ICH rats; and STZ rats vs. STZ rats with ICH, $\mathrm{n}=8-10$ in each group). In addition, Fig. 4 shows that ICV infusion of ML228 significantly augmented the levels of VEGF in the brain tissues of nonSTZ rats and STZ rats after ICH.

\section{Expression of VEGFR-2 and Caspase-3}

Figure $5 \mathrm{~A}$ illustrates that induction of ICH significantly increased the protein expression of VEGFR-2 in the brain tissues of non-STZ rats and STZ rats $(P<0.05$, control rats vs. ICH rats; and STZ-rats vs. STZ-rats with ICH, $\mathrm{n}=8-12$ in each group). However, the augmented VEGFR-2 
Fig. 3. ICH significantly elevated the levels of VEGF in the brain tissues of non-STZ rats and STZ rats. The increases were seen 1, 3 and 7 days after induction of ICH and greater increases appeared 3 days after ICH. Data are expressed as mean \pm SEM. ${ }^{*} P<0.05$, indicated control rats $v s$. ICH rats; and STZ rats vs. STZ rats with $\mathrm{ICH}(\mathrm{n}=10$ 12 in each group). $\dagger P<0.05$ vs. non-STZ rats with ICH.
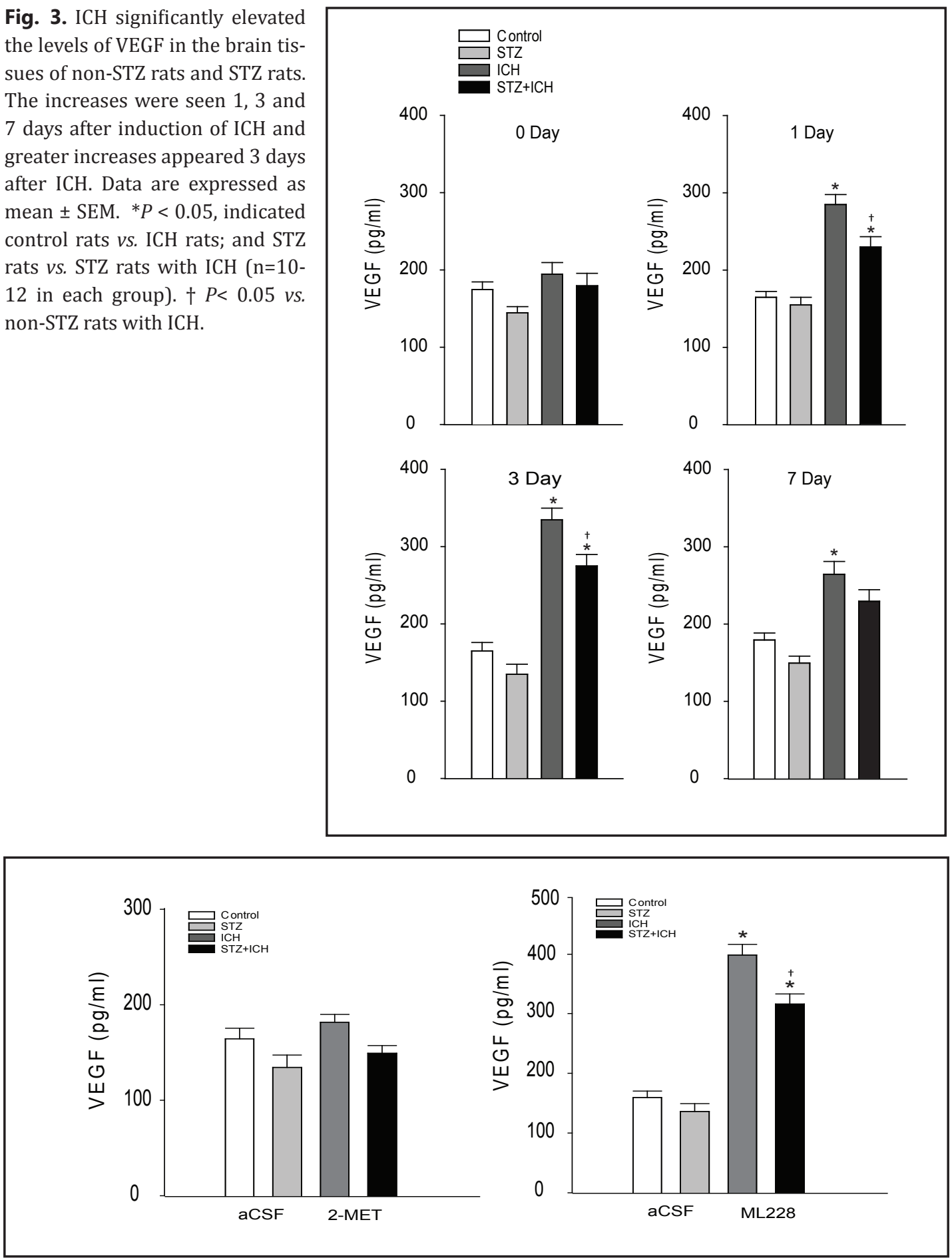

Fig. 4. ICV infusion of 2-MET $(100 \mu \mathrm{g})$ attenuated increases of VEGF evoked by ICH in non-STZ rats and STZ rats with ICH (n=8-10 in each group). Likewise, ICV infusion of ML228 (100 $\mu$ g) significantly augmented the levels of VEGF in the brain tissues of non-STZ rats and STZ rats after ICH. ${ }^{*} P<0.05$, control rats vs. ICH rats; and STZ rats vs. STZ rats with ICH (n=8-10 in each group). $† P<0.05$ vs. non-STZ rats with ICH.

was smaller in STZ rats than that in non-STZ rats 3 days after ICH. As ML228 was infused into the brain to stabilize HIF-1 $\alpha$, VEGFR-2 expression was enhanced and the increased levels were attenuated in STZ rats as compared with non-STZ rats. Moreover, Fig. 5B shows that 
A
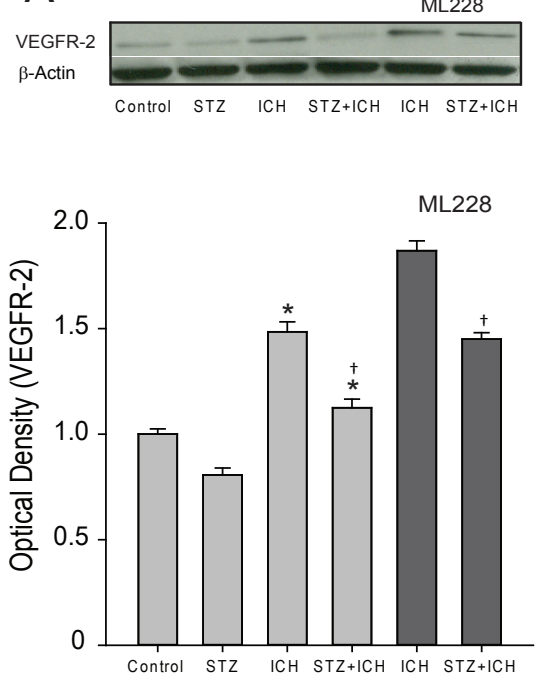

C

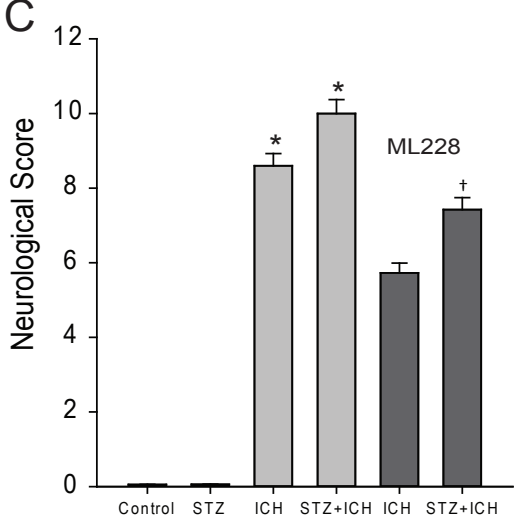

B

Caspase-3

$\beta$-Actin
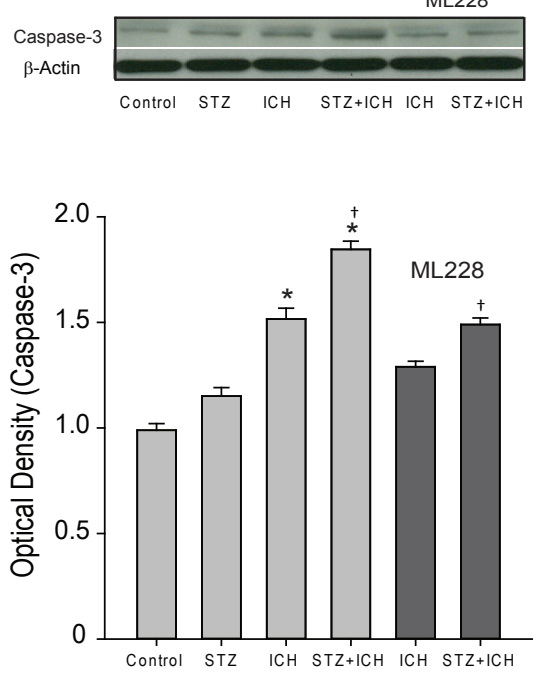

$D_{90}$

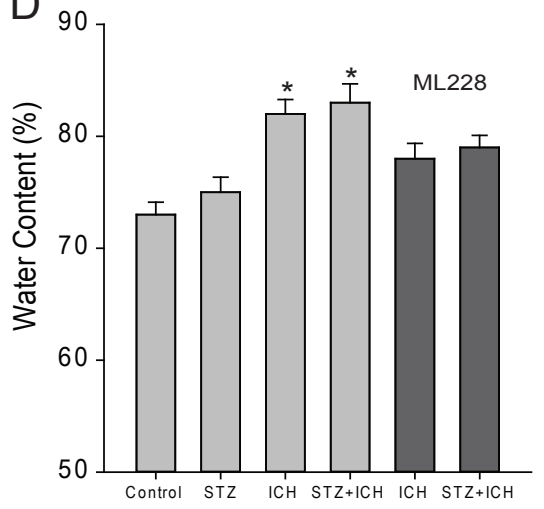

Fig. 5. (A). ICH significantly increased the protein expression of VEGFR-2 in the brain tissues of non-STZ rats and STZ rats. However, the augmented VEGFR-2 was smaller in STZ rats than that in non-STZ rats 3 days

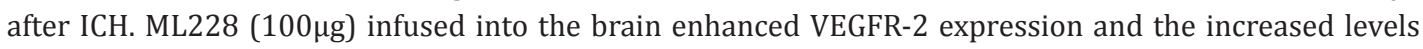
were attenuated in STZ rats as compared with non-STZ rats. (B). ICH significantly increased expression of Caspase-3 in both non-STZ rats and STZ rats. An increase in Caspase-3 was greater in STZ rats than in nonSTZ rats. ICV infusion of ML228 attenuated expression of Caspase- $3 .{ }^{*} \mathrm{P}<0.05$, control rats vs. ICH rats; and STZ-rats vs. STZ-rats with ICH ( $\mathrm{n}=8-12$ in each group). $\uparrow \mathrm{P}<0.05$ vs. non-STZ rats with ICH. (C) \& (D). ICH increased the mNSS and water content of brain tissues in non-STZ rats and STZ rats. ML228 attenuated the increased nNSS and water content in both groups of animals and the effects on the mNSS were greater in non-STZ rats compared with STZ rats. ${ }^{*} \mathrm{P}<0.05$ vs. non-STZ and STZ rats without ICH and rats with treatment of ML228 ( $n=10-12$ in each group). $\dagger \mathrm{P}<0.05$ vs. non-STZ rats with ICH.

ICH significantly increased expression of Caspase-3 in both non-STZ rats and STZ rats. Note that the increased Caspase-3 was greater in STZ rats than in non-STZ rats. Also, ICV infusion of ML228 significantly attenuated expression of Caspase-3.

\section{Neurological Function and Brain Edema}

Figure 5C demonstrates that ICH led to increases in the mNSS in non-STZ rats and STZ rats $(P<0.05$, control rats vs. ICH rats; and STZ rats vs. STZ rats with ICH, $\mathrm{n}=10-12$ in each group). Also, this figure shows that ML228 significantly attenuated the increased nNSS in both groups of animals and the effects were observed to be greater in non-STZ rats compared 
with STZ rats. Similarly, Fig. 5D shows that induction of ICH amplified water content of brain tissues in non-STZ rats and STZ rats and infusion of ML228 significantly attenuated increases of water content.

\section{Discussion}

A rat model of ICH was widely employed to determine the mechanisms responsible for neurological damages. In the current study, using the same rat model our data showed that HIF- $1 \alpha$ was significantly increased in the brain tissues of non-STZ rats and STZ rats 1-7 days after induction of ICH (Fig. 2). Also, brain VEGF was increased and time courses for increases of HIF- $1 \alpha$ and VEGF were similar (Fig. 3). Caspase-3 as an indicator of neuronal apoptosis was also examined in this study. Our data further demonstrated that ICH increased the levels of VEGFR-2 and Caspase-3 in the brain tissues (Fig. 5A\&B) in non-STZ rats and STZ rats. Interestingly, our findings showed that ICH-evoked HIF- $1 \alpha$ and VEGF were less in STZ rats as compared with non-STZ rats, suggesting that the responses of HIF- $1 \alpha$ and VEGF to ICH were attenuated under the diabetic hyperglycemia. Also, upregulated VEGFR-2 was impaired in STZ rats. ICV infusion of ML228 to stabilize HIF- $1 \alpha$ in the brain tissues significantly augmented VEGFR-2 and attenuated Caspase-3 to a less degree in STZ-rats than in non-STZ rats (Fig. $5 A \& B)$. In consistent with this result, our data showed that damaged neurological scores and increased water content caused by ICH were significantly improved by ML228. Overall, we suggest that activated HIF- $1 \alpha$ likely plays a beneficial role in improving neurological deficits induced by ICH via VEGF mechanisms and the response of HIF- $1 \alpha$ to ICH is largely impaired with diabetic hyperglycemia. Also, stabilizing HIF- $1 \alpha$ improves expression of VEGFR-2 and attenuates Caspase- 3 and this is likely to have improving effects on neurological deficits observed after induction of ICH.

Previous studies showed that the high expression of VEGF in the brain tissues has protective effects against ICH by improving the permeability of blood brain barrier, reducing brain edema formation and promoting the recovery of brain injuries [20]. In our study, a group of experiment was designed to examine if the effects of HIF- $1 \alpha$ on VEGF levels (Fig. 4). We observed that infusion of ML228 increased VEGF evoked by ICH in both non-STZ rats and STZ animals. This suggests that ICV of this dosage of ML228 was effective. Also, the data suggest the underlying mechanisms that the role of ML228 observed in our current study in alleviating the ICH injuries is likely due to the up-regulation of VEGF, which may further enhance the production of new blood vessels and microvessels and increase oxygen supply to the hematomal brain tissues, significantly improving the ischemia and hypoxia of brain tissue and nerve cells.

In addition, apoptosis represents a prominent form of cell death and observed in the brain tissues after ICH. Neuronal apoptosis result in blood brain barrier dysfunction, inflammation and oxidative cascades and thereby leading to brain damage [23, 24]. It has been reported that Caspase- 3 expression was exaggerated in cortical neurons after subarachnoid hemorrhage and inhibition of Caspase- 3 reduces the neuron loss and brain edema [24, 25]. Our current study showed that activated Caspase- 3 by ICH was significantly attenuated by ICV infusion of ML288 stabilizing HIF-1 $\alpha$ levels after induction of ICH.

Strong evidence suggests the activation of ERK-MAPK signaling pathway is engaged in the high levels of HIF- $1 \alpha$ mediating the ICH-induced neuronal injury because the inhibition of MAPK decreases brain water content, suppression of hematoma expansion and edema formation $[26,27]$. JNK1/2 is commonly activated in inflammatory tissues and recognized as one of central signaling molecules in regulating brain ischemic injuries $[28,29]$. The activation of JNK and p38 MAPK signal pathways likely increases the expression of VEGF [30]. A recent report suggests that Urocortin has the anti-inflammatory and neuroprotective effects via the phosphorylation of p38 and JNK1/2 and thereby increasing VEGF expression in animal model of ICH [31]. Results of this prior study also showed engagement of these 
Yu et al.: HIF-1 $\alpha$ Activated by Intracerebral Hemorrhage in Diabetes

signaling pathways contributes to protective the effects of Urocortin on ICH inducedneurological functional deficits and brain edema [31].

In diabetes models, these signaling pathways are altered and this further leads to worsen neurological deficits $[5,8]$. These findings are consistent with results observed in patients with diabetes since hyperglycemia is a strong risk factor for poor outcome after stroke $[6,7]$. Nevertheless, diabetes is a condition of accelerated vascular disorders. Our current study offers the first evidence that enhancement of HIF- $1 \alpha$ and VEGF after induction of ICH are impaired and thereby leads to worsen VEGFR-2 and Caspase-3 expression in animals with diabetic hyperglycemia. Also, our results suggest that cerebral administration of HIF-1 activator improves the levels of VEGFR-2 and Caspase- 3 and brain injury induced by ICH.

\section{Conclusions}

During ICH, activation of HIF- $1 \alpha$ is likely to play a beneficial role in modulating neurological dysfunction via VEGF mechanisms and response of HIF- $1 \alpha$ is largely impaired in diabetes. Results of this study will have pharmacological implications to target specific HIF$1 \alpha$ and its pathway VEGF for neural disorder and vulnerability related to ICH. Particularly, our data provide evidence that HIF-1 $\alpha$ and VEGF contribute to brain injury often seen in diabetic patients.

\section{Acknowledgments}

This study was supported by grant from the Natural Science Foundation of Chongqing (Grant\#. CSTC2012JJA10067).

\section{Disclosure Statement}

None.

\section{References}

1 van Asch CJJ, Luitse MJA, Rinkel GJE, van der Tweel I, Algra A, Klijn CJM: Incidence, case fatality, and functional outcome of intracerebral haemorrhage over time, according to age, sex, and ethnic origin: a systematic review and meta-analysis. Lancet Neurol 2010;9:167-176.

2 Hoff J, Xi G. Brain edema from intracerebral hemorrhage. Acta Neurochir Suppl 2003;86:11-15.

3 Rincon F, Mayer SA: Novel therapies for intracerebral hemorrhage. Curr Opin Crit Care 2004;10:94-100.

4 Hill MD: Stroke and diabetes mellitus. Handb Clin Neurol 2014;126:167-174.

5 Fan X, Jiang Y, Yu Z, Yuan J, Sun X, Xiang S, Lo E, Wang X: Combination approaches to attenuate hemorrhagic transformation after tPA thrombolytic therapy in patients with poststroke hyperglycemia/diabetes. Adv Pharmacol 2014;71:391-410.

6 Tapia-Perez JH, Gehring S, Zilke R, Schneider T: Effect of increased glucose levels on short-term outcome in hypertensive spontaneous intracerebral hemorrhage. Clin Neurol and Neurosurg 2014;118:37-43.

7 Zhang G, Wu F, Xu Y, Feng J, Cai Z, Xu B, Zhou X, He M: Prestroke glycemic status is associated with the functional outcome in spontaneous intracerebral hemorrhage. Neurol Sci 2015; 36:927-934.

8 Ding G, Yan T, Chen J, Chopp M, Li L, Li Q Cui C, Ning R, Jiang Q: Persistent cerebrovascular damage after stroke in type two diabetic rats measured by magnetic resonance imaging. Stroke 2015;46:507-512. 


\section{Cellular Physiology Cell Physiol Biochem 2015;37:67-76 and Biochemistry DOI: 10.1159/000430334 $\quad$ (c) 2015 S. Karger AG, Base \\ Yu et al.: HIF-1 $\alpha$ Activated by Intracerebral Hemorrhage in Diabetes}

9 Xu S, Wu Q, Guo G, Ding X: The protective effects of urocortin1 against intracerebral hemorrhage by activating JNK1/2 and p38 phosphorylation and further increasing VEGF via corticotropin-releasing factor receptor 2. Neurosci Lett 2015;589:31-36.

10 Ceradini DJ, Kulkarni AR, Callaghan MJ, Tepper OM, Bastidas N, Kleinman ME, Capla JM, Galiano RD, Levine JP, Gurtner GC: Progenitor cell trafficking is regulated by hypoxic gradients through HIF-1 induction of SDF1. Nat Med 2004;10:858-864.

11 Manalo DJ, Rowan A, Lavoie T, Natarajan L, Kelly BD, Ye SQ, Garcia JG, Semenza GL: Transcriptional regulation of vascular endothelial cell responses to hypoxia by HIF-1. Blood 2005;105:659-669.

12 Sun Y, Li QF, Zhang Y, Hu R, Jiang H: Isoflurane preconditioning increases survival of rat skin randompattern flaps by induction of HIF-1alpha expression. Cell Physiol Biochem 2013;31:579-591.

13 Wang P, Qi H, Sun C, He W, Chen G, Li L, Wang F: Overexpression of hypoxia-inducible factor-1alpha exacerbates endothelial barrier dysfunction induced by hypoxia. Cell Physiol Biochem 2013;32:859-870.

14 An Y, Zhang JZ, Han J, Yang HP, Tie L, Yang XY, Xiao Y, Pan Y, Li XJ: Hypoxia-inducible factor-1alpha dependent pathways mediate the renoprotective role of acetazolamide against renal ischemia-reperfusion injury. Cell Physiol Biochem 2013;32:1151-1166.

15 Hota KB, Hota SK, Srivastava RB, Singh SB: Neuroglobin regulates hypoxic response of neuronal cells through Hif-1[alpha]- and Nrf2-mediated mechanism. J Cereb Blood Flow Metab 2012;32:1046-1060.

16 Liu Y, Cui Y, Shi M, Zhang Q, Wang Q, Chen X: Deferoxamine promotes MDA-MB-231 cell migration and invasion through increased ROS-dependent HIF-1alpha accumulation. Cell Physiol Biochem 2014;33:10361046.

17 Northington FJ, Chavez-Valdez R, Martin LJ: Neuronal cell death in neonatal hypoxia-ischemia. Ann Neurol 2011;69:743-758.

18 Kim Y-W, Byzova TV: Oxidative stress in angiogenesis and vascular disease. Blood 2014;123:625-631.

19 Zhou HJ, Tang T, Cui HJ, Yang AL, Luo JK, Lin Y, Yang QD, Li XQ: Thrombin-triggered angiogenesis in rat brains following experimental intracerebral hemorrhage. J Neurosurg 2012;117:920-928.

20 Peng Z-R, Yang AL, Yang Q-D: The effect of hyperbaric oxygen on intracephalic angiogenesis in rats with intracerebral hemorrhage. J Neurol Sci 2014;342:114-123.

21 Xu G-Y, Li G, Liu N, Huang L-Y: Mechanisms underlying purinergic P2X3 receptor mediated mechanical allodynia induced in diabetic rats. Mol Pain 2011;7:60.

22 Zhang Y, Yi B, Ma J, Zhang L, Zhang H, Yang Y, Dai Y: Quercetin promotes neuronal and behavioral recovery by suppressing inflammatory response and apoptosis in a rat model of intracerebral hemorrhage. Neurochem Res 2015;40:195-203.

23 Bosoi CR, Yang X, Huynh J, Parent-Robitaille C, Jiang W, Tremblay M, Rose CF: Systemic oxidative stress is implicated in the pathogenesis of brain edema in rats with chronic liver failure. Free Radical Biol Med 2012;52:1228-1235.

24 Simard J, Geng Z, Kyoon Woo S, Ivanova S, Tosun C, Melnichenko L, Gerzanich V: Glibenclamide reduces inflammation, vasogenic edema, and caspase-3 activation after subarachnoid hemorrhage. J Cereb Blood Flow Metab. 2008;29:317-330.

25 Dong YS, Wang JL, Feng DY, Qin HZ, Wen H, Yin ZM, Gao GD, Li C: Protective effect of quercetin against oxidative stress and brain edema in an experimental rat model of subarachnoid hemorrhage. Int J Med Sci 2014;11:282-290.

26 Fujimoto S, Katsuki H, Ohnishi M, Takagi M, Kume T, Akaike A: Thrombin induces striatal neurotoxicity depending on mitogen-activated protein kinase pathways in vivo. Neurosci 2007;144:694-701.

27 Ohnishi M, Katsuki H, Fujimoto S, Takagi M, Kume T, Akaike A: Involvement of thrombin and mitogenactivated protein kinase pathways in hemorrhagic brain injury. Exp Neurol 2007;206:43-52.

28 Wan S, Zhan R, Zheng S, Hua Y, Xi G: Activation of c-Jun-N-terminal kinase in a rat model of intracerebral hemorrhage: The role of iron. Neurosci Res 2009;63:100-105.

29 Wang X-T, Pei D-S, Xu J, Guan Q-H, Sun Y-F, Liu X-M, Zhang GY: Opposing effects of Bad phosphorylation at two distinct sites by Akt1 and JNK1/2 on ischemic brain injury. Cell Signal 2007;19:1844-1856.

30 Pagès G, Berra E, Milanini J, Levy AP, Pouysségur J: Stress-activated Protein Kinases (JNK and p38/HOG) Are Essential for Vascular Endothelial Growth Factor mRNA Stability. J Biol Chem 2000;275:26484-26491.

31 Xu S, Wu Q, Guo G, Ding X: The protective effects of urocortin1 against intracerebral hemorrhage by activating JNK1/2 and p38 phosphorylation and further increasing VEGF via corticotropin-releasing factor receptor 2. Neurosci Lett 2015;589:31-36. 